\title{
Principle and design of a mobile arm support for people with muscular weakness
}

\author{
Just L. Herder, PhD; ${ }^{1 *}$ Niels Vrijlandt, MSc; ${ }^{2}$ Tonko Antonides, MSc; ${ }^{2}$ Marijn Cloosterman, BSc; ${ }^{2}$ \\ Peter L. Mastenbroek, BSc ${ }^{2}$ \\ ${ }^{1}$ Department of Biomechanical Engineering, Delft University of Technology, Mekelweg, the Netherlands; \\ ${ }^{2}$ Microgravity Products BV, Van Nelleweg, Rotterdam, the Netherlands
}

\begin{abstract}
This article describes the development of a mobile arm support for people with muscular diseases. The arm support is spring-balanced, with special attention on reduction of operating effort (high balancing quality and low friction), functionality (large range of motion), and aesthetics (inconspicuous design). The spring settings can be adjusted for wearing heavier clothing or picking up an object, a function that can also be used for moving up or down. The device levels itself automatically to compensate for uneven floors, a function that can be overruled to assist forward/backward motion of the arm. Thus, the balancer can compensate for the weight of the arm and be adjusted to generate force to a limited (safe) extent. The principle and design of the mechanism are presented and preliminary field trial results are given. Two users report on 6 months of continuous use of the arm support in their home and social environments.
\end{abstract}

Key words: adjustable spring mechanism, assistive device, biomechanics, gravity equilibrator, mobile arm support, neuromuscular diseases, passive orthosis, rehabilitation, static balancing, upper limb, user opinions.

\section{INTRODUCTION}

People suffering from neuromuscular diseases have trouble lifting their arms against gravity, although a large number of them maintain sensitivity and residual strength in their hands. Therefore, a device is desired that enables them to use their hands in a larger range of motion (ROM) than they can reach themselves. This is particu- larly true for patients with spinal muscular atrophy (SMA), a disease having an incidence in the range of 4 per 100,000 [1]. In this disease, the proximal joints (shoulders, hips) are affected first. Over time, performing basic activities of daily living (ADL) unassisted becomes increasingly difficult. For patients, this leads to a feeling of reduced independence.

Available assistive devices can be subdivided in three main groups that are mentioned next with some illustrations [2]. First, a number of rehabilitation robotic manipulators have been developed. Some have been successfully commercialized [3], including the Massachusetts Institute of Technology (MIT)-Manus, the Handy, and the Raptor. Powered orthoses make up the second group; for example, the exoskeletons Motorized Upper Limb Orthotic System (MULOS) [4] and the Golden Arm [5]; the active overhead suspension presented by Homma and Arai [6] should also be included in this group. A third group is

Abbreviations: ADL = activities of daily living, $\mathrm{CCM}=$ combined center of mass, DOF = degrees of freedom, MAS = mobile arm support, MULOS = Motorized Upper Limb Orthotic System, MGP = Microgravity Products, ROM = range of motion, SMA = spinal muscular atrophy.

*Address all correspondence to Just L. Herder, PhD; Department of Biomechanical Engineering, Delft University of Technology, Mekelweg 2, 2628 CD Delft, the Netherlands; +31-15-278-4713; fax: +31-15-278-4717.

Email: j.l.herder@3me.tudelft.nl

DOI: 10.1682/JRRD.2006.05.0044 
composed of nonpowered orthoses, typically based on static balancing using springs. Chyatte and Vignos [7] and Skorecki [8] are two of the earliest with current efforts being made by Rahman et al. [9]. The JAECO mobile arm support (MAS) (JAECO Orthopedic, Hot Springs, Arkansas) [10] and the TOP-HELP (Focal Revalidatie-technik, Berkel-Enschot, the Netherlands) [11] are two examples of commercially available nonpowered orthoses.

Robotic manipulators and powered orthoses are intended for the weakest patients, who in some cases have virtually no muscle force. If the user can be classified according to Brooke [12] in categories 3 to 5, a passive arm orthosis is usually preferred [13]. Passive (nonpowered) orthoses require some muscle force for accelerating and decelerating and for overcoming friction and balancing errors. Moreover, a changing load due to picking up objects or changing clothing are not considered and therefore need to be carried by muscle force. In particular, the effort for a change of clothing can be substantial and disqualifies nonpowered orthoses for many patients. Most currently available passive arm supports cannot be adjusted by the user. In addition, some suffer from limited ROM (e.g., only horizontal), nonperfect balancing quality (e.g., due to rubber springs), or problems related to comfort (donning and doffing, sliding and perspiration in trough). Therefore, the need still exists for an arm support that acts with satisfying functionality, comfort, safety, and aesthetics.

In this article, we propose a passive arm support design that reduces the operating effort associated with nonpowered orthoses by striving for low friction and zero balancing error, while at the same time aiming at high functionality (ROM) and aesthetics. Our study is primarily directed at persons with SMA, although the result has a much wider application potential, including persons with other neuromuscular diseases (e.g., multiple sclerosis, Becker, Shoulder Girdle), persons with certain paralyses, and persons performing computer work or general desk tasks who have or are at risk for repetitive strain injury.

\section{METHODS}

\section{Design Specifications}

From literature [9,14-16] and previous work [2], we determined desired functionality and the corresponding required ROM [13]. Herder reports that the device should aid important ADL such as feeding oneself, personal hygiene (touching face, head), and reaching (to grasp objects and move them over to the lap or wheelchair table, to reach keyboard and other things on tables) [13].

From home visits with three users, Cardoso et al. found that key factors for a useful orthosis are an inconspicuous appearance, comfortable in different circumstances (clothing), and easy operation (low mental and physical effort). Also, they found that the fixed armrest may not be sacrificed in favor of a MAS, because it is essential for trunk balance [2]. Finally, users generally preferred a nonpowered device because this concept inherently uses the natural control still present, it tends to be less conspicuous, and low energy consumption is a vital issue, especially for those persons using respiration augmentation.

Cardoso et al. stated that the design should meet the following quantitative requirements. We performed a rough investigation in our target group using a sling and spring scales that revealed that the heaviest user arms weigh approximately $30 \mathrm{~N}$, excluding clothing and picked-up objects. We therefore set the upper bound for the support force to be generated at $35 \mathrm{~N}$. We set the maximum allowable error from balancing inaccuracy or friction at $1 \mathrm{~N}$. As to the ROM, Cardoso et al. had reported that the device should be able to bring the hand of the user to the mouth (feeding), face (hygiene), and head (combing hair), as well as across the lap or wheelchair table. Furthermore, users wanted to be able to reach the work top of desks, tables, and kitchen sinks. In addition, qualitative requirements were defined, mainly relating to comfort and inconspicuousness. Finally, the fixed wheelchair armrest was to be maintained.

\section{Biomechanical Working Principle}

From design specifications, we concluded that the technology of static balancing would be useful. We considered a previously developed mechanism for balancing the patient's arm. This mechanism, called "Anthropomobile Robot Arm," is a two-segment open chain with 4 degrees of freedom (DOF), statically balanced by two zero-free-length springs [17]. With the same mobility as the human arm, it seemed well suited to be placed alongside the user's arm. Such a design would yield the most compact mechanism with few singularities and little risk of interference with the wheelchair or the user. However, its drawbacks are that it (1) is conspicuous (wearing it underneath clothing is unfeasible because it needs to be 
mounted to the wheelchair), (2) causes discomfort around the shoulder (e.g., a complex construction with a virtual joint in the shoulder, such as in the MULOS, or a joint next to the shoulder resulting in sliding along the arm), (3) requires two interfaces (one on the forearm and one on the upper arm), and (4) only works well if the shoulder is in a specified (fixed) position [2]. Consequently, we found this track conceptually was not a satisfactory design.

A review of the force analysis led us to another solution principle [2]. Figure 1(a) shows a free-body diagram of the upper arm. To equilibrate the upper arm mass $m_{1}$,

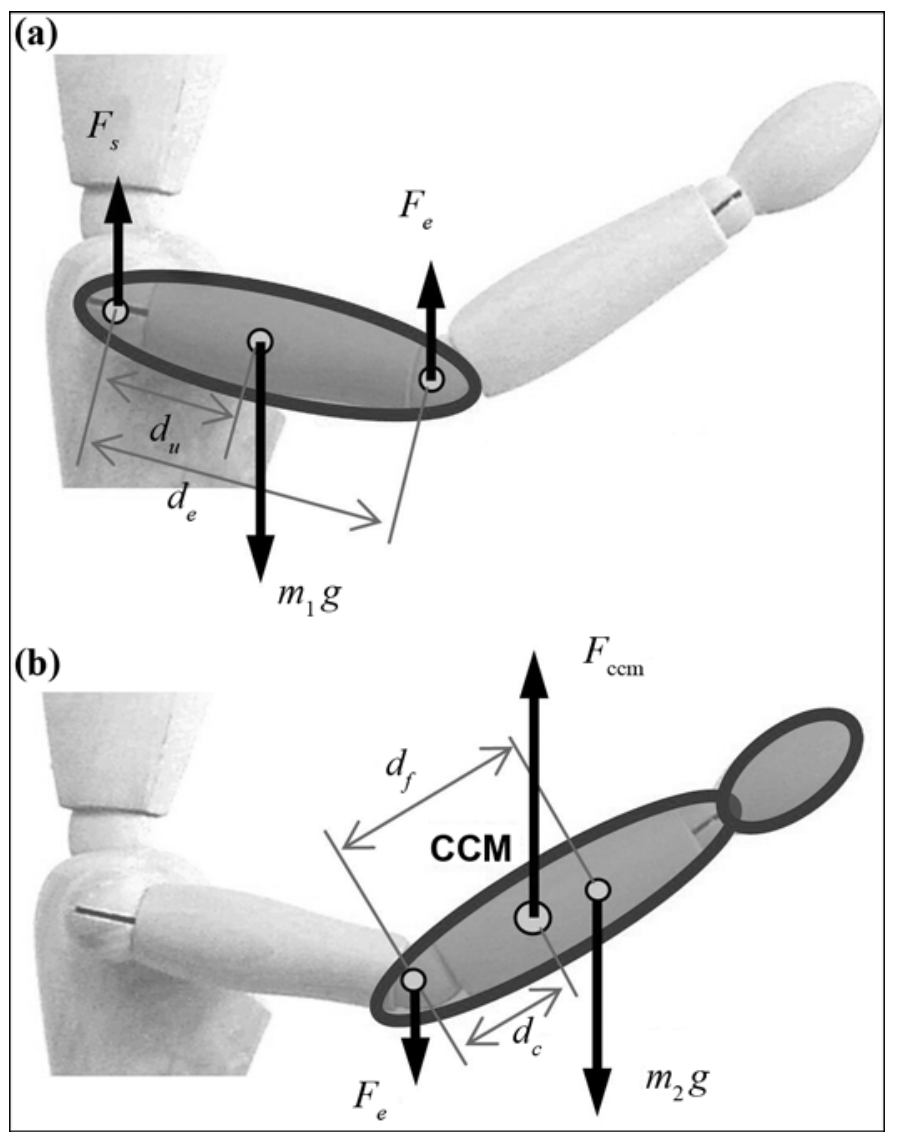

Figure 1.

Free-body diagrams of arm segments: (a) upper arm and (b) forearm. If shoulder carries force $F_{s}$, then elbow force $F_{e}$ can be transferred to forearm so that only single vertical constant support force $F_{\mathrm{ccm}}$ is required to statically balance user's arm. $d_{c}=$ distance from elbow to combined center of mass (CCM), $d_{e}=$ distance from shoulder joint to elbow joint, $d_{f}=$ distance from elbow joint to center of mass of forearm and hand combined, $d_{u}=$ distance from shoulder joint to center of mass of upper arm, $g$ = acceleration of gravity, $m_{1}=$ mass of upper arm, $m_{2}=$ mass of forearm and hand. one could employ an interface with the support mechanism. However, this is not required if one observes that the upper arm can also be equilibrated by two forces: $F_{S}$ in the shoulder and $F_{e}$ in the elbow, where $F_{e}=m_{1} g d_{u} / d_{e}$ and $F_{s}=m_{1} g\left(1-d_{u} / d_{e}\right.$ ) (where $g=$ acceleration of activity, $d_{u}=$ distance from shoulder to center of mass of upper arm, and $d_{e}=$ distance from shoulder to elbow joint). The patient's shoulder joint can carry about half of the upper arm mass $\left(d_{u} \approx 0.5 d_{e}\right)$. The other half is transferred to the forearm. Figure 1(b) shows a free-body diagram of the forearm (and hand), including the reaction of $F_{e}$, which together with the forearm mass constitute the load on this subsystem. Interestingly, these two forces are constant and can be combined into one constant force that applies at the combined center of mass (CCM) (Figure 1(b)). Therefore, if a vertical constant support force $F_{\mathrm{ccm}}=\left(m_{1} d_{u} / d_{e}+m_{2}\right) g$ is provided in any configuration of the mechanism, then the user's arm will be perfectly statically balanced in all its DOF, even though only about 75 percent of the patient's arm mass $\left(m_{1}\right.$ and $m_{2}$ being roughly equal, where $m_{2}=$ mass of forearm and hand) is actually supported by the orthosis (the remainder being carried by the shoulder). Moreover, only one point (the CCM) needs to be supported, as opposed to the initial design, where two interfaces were required [17]. Consequently, the mechanism no longer needs to be arranged alongside the arm. Other designs also employ a single interface $[4,7,9]$, but this biomechanical analysis was what produced the following design concept.

Since just one point needs to be supported, we gained great design latitude and found many gravity balancers to qualify for this task. One seemed particularly suitable, namely, a spring-loaded parallelogram mechanism (Figure 2) [18-20]. Kinematically, the mechanism is a hybrid version of a serial (open-loop kinematic chain) and a parallel (closed kinematic chain) mechanism, combining the advantages of a large ROM (serial) and all springs close to the base (parallel). The linkage consists of a base link with auxiliary parallelogram and a final link attaching to the interface, and it moves in a vertical plane by revolute joints perpendicular to the plane. The longer side of the auxiliary parallelogram is parallel to the base link; the shorter side is parallel to the final link. The parallelogram thus moves with two DOF. Two separate springs are required, one for each DOF. The plane of motion is rotatable about the vertical through a fixed pivot. Thus, $3 \mathrm{DOF}$ were obtained so that the endpoint of the mechanism can follow the CCM, where it is connected to the user's arm 


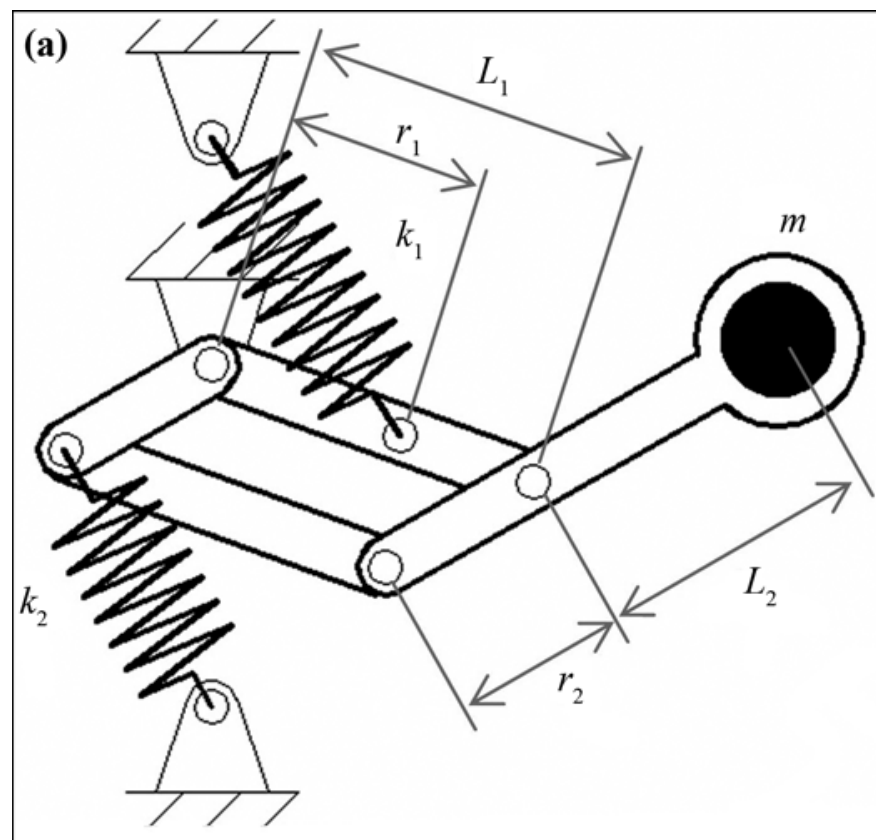

(b)

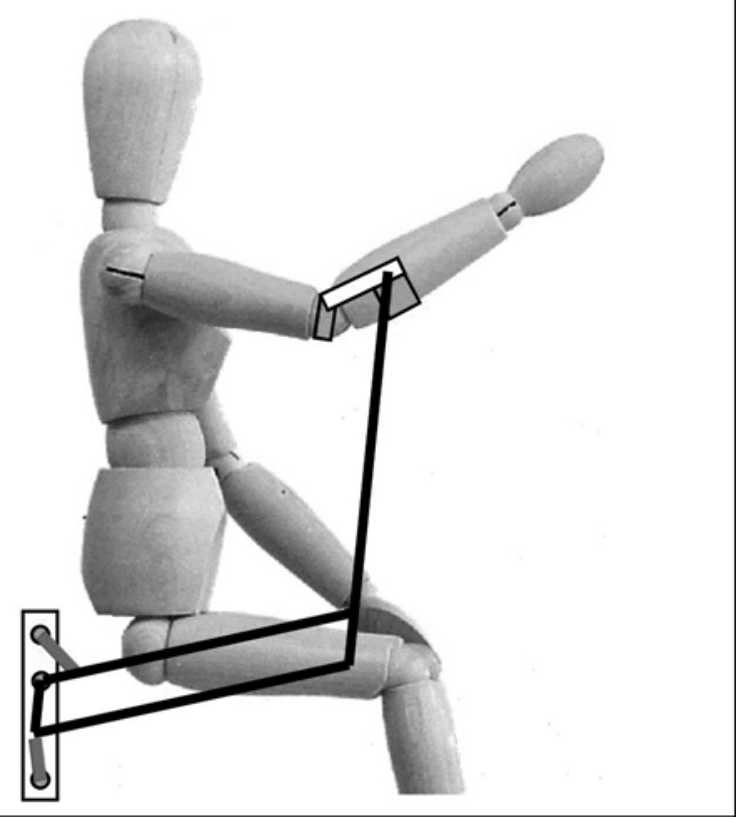

Figure 2.

Parallelogram linkage and balancer design used for first prototype: (a) diagram showing parameters and (b) diagram showing use as arm support. $m=$ supported mass, $k_{1}=$ stiffness of proximal link spring, $k_{2}=$ stiffness of distal link spring, $L_{1}=$ proximal link length, $L_{2}=$ distal link length, $r_{1}=$ proximal spring attachment arm length, $r_{2}=$ distal spring attachment arm length.

through an interface. The connection mechanism to the interface contains two additional rotational DOF to allow for different orientations of the forearm relative to the mechanism. A first joint allows rotation about an essentially horizontal axis, perpendicular to the final link. In addition, an essentially vertical axis is located immediately underneath the interface, which has a limited ROM - enough to provide sufficient rotation of the forearm in the horizontal plane (the main movement comes from the vertical base joint) and to avoid interference between elbow and final link.

We created a prototype and conducted home visits with three users. All three users could lift their arms against gravity (Figure 3) and found the concept promising. In particular, users appreciated the aesthetics (the device is compact, placed below the fixed armrest, and is hidden to the user except for the interface and part of the final link), the intuitive control (no joysticks or similar required), and the natural feel. This result demonstrated that the CCM principle works well, i.e., the shoulder may be used to carry part of the upper-arm weight. Furthermore, the prototype was useful for generating user feedback on the functionality of the device. Based on these results, we designed, manufactured, and tested a second prototype (Figure 4) [13]. This article focuses on the third and final design iteration.

\section{Final Design}

Based on the results of the first and second prototypes, we identified five areas of improvement: (1) optimization of linkage design to eliminate interference and to minimize the link lengths, (2) optimization of balancing quality and adjustment of the balancer, (3) improvement of the interface in comfort and ease of donning and doffing, (4) general improvement of appearance, and (5) ease of use.

\section{Optimization of Linkage Design}

To avoid the interference with the wheelchair and its armrest, we modified the parallelogram mechanism. In fact, as compared with the first prototype, we selected the other branch (i.e., solution of inverse kinematics), where the two parallel links are extending up, over the armrest, and the end link extends forward to the interface with the user's arm (Figure 5). We preferred a fulcrum location below the fixed armrest, although we disqualified a location immediately below the shoulder because of the singularities on this vertical line. This left the link lengths as the main parameters to optimize. We found the resulting minimum link lengths to be $280 \mathrm{~mm}$ for the base link $\left(L_{2}\right)$ and $320 \mathrm{~mm}$ for the final link $\left(L_{1}\right)$ [13]. In the final design, we increased the base link length to $320 \mathrm{~mm}$ to accommodate the springs. We incorporated a curve in the final link to 


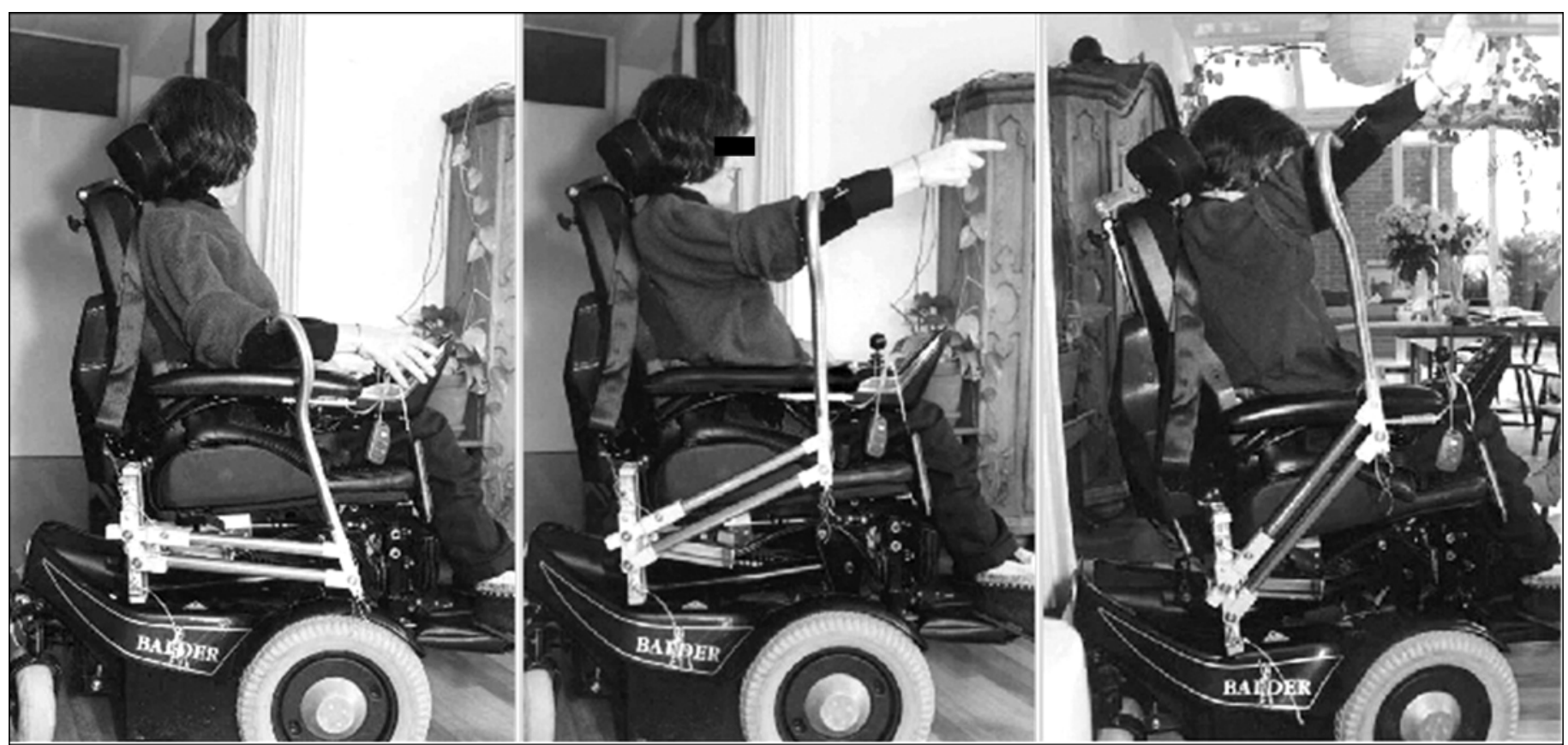

Figure 3.

User (spinal muscular atrophy patient) demonstrating her increased range of motion with first arm support prototype.

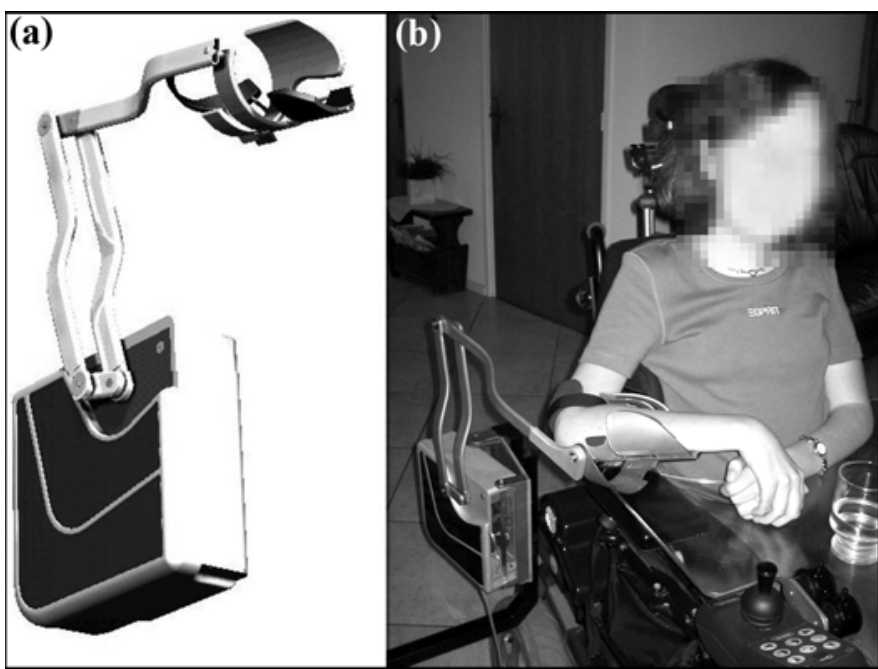

Figure 4.

Second arm support prototype: (a) computer-aided drawing showing interface, parallelogram linkage, and box containing spring mechanism and (b) device in evaluation test.

avoid interference with the user's elbow. The workspace of the mechanism can be characterized as follows: from a vertical position, the base link can rotate $45^{\circ}$ backward and $25^{\circ}$ forward. The final link can extend, from a position perpendicular to the base link, at most, $50^{\circ}$ up and $50^{\circ}$ down, when the base link is vertical. If the base link is rotated forward, the range of moving up of the final link is reduced by the rotation angle of the base link. If the base link is rotated back, the range of downward motion of the final link is reduced by the rotation angle of the base link.

\section{Optimization of Balancing Quality and Adjustment}

With the change in mechanism kinematics, we also redesigned the balancing mechanism, while accounting for the desire for adjustability. The linkage can be balanced by springs that are all located around the base joint of the mechanism (Figure 5). A theoretically perfect balancing quality can be obtained with springs in which the force is proportional to their total length, rather than to its elongation (i.e., free length is zero). This spring behavior can be achieved in various ways, including increased initial tension, pulley-and-string arrangements, and special constructions [20]. With these springs, the balancing conditions for the linkage are $[2,20]$

$$
\begin{aligned}
& m g L_{1}=k_{1} a_{1} r_{1}, \\
& m g L_{2}=k_{2} a_{2} r_{2},
\end{aligned}
$$

where $a_{i}$ is the fixed spring arm, $r_{i}$ is the spring arm on the moving link, $L_{i}$ is the link length, and $k_{i}$ is the spring stiffness. In these equations, link mass is neglected for simplicity of presentation. Link mass can be incorporated without 


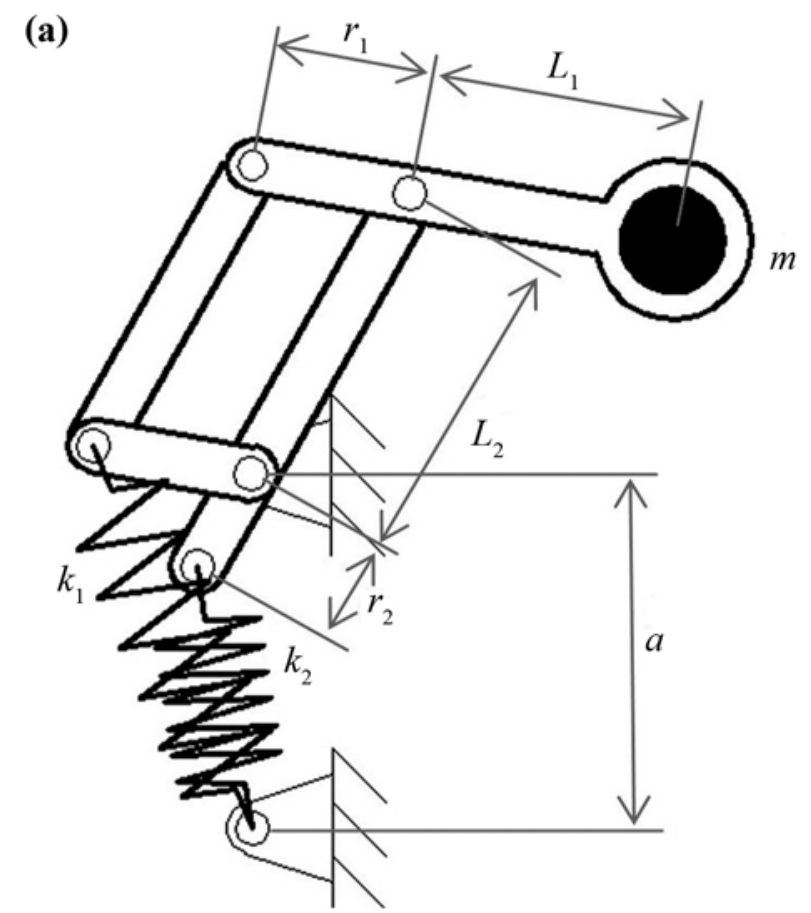

(b)

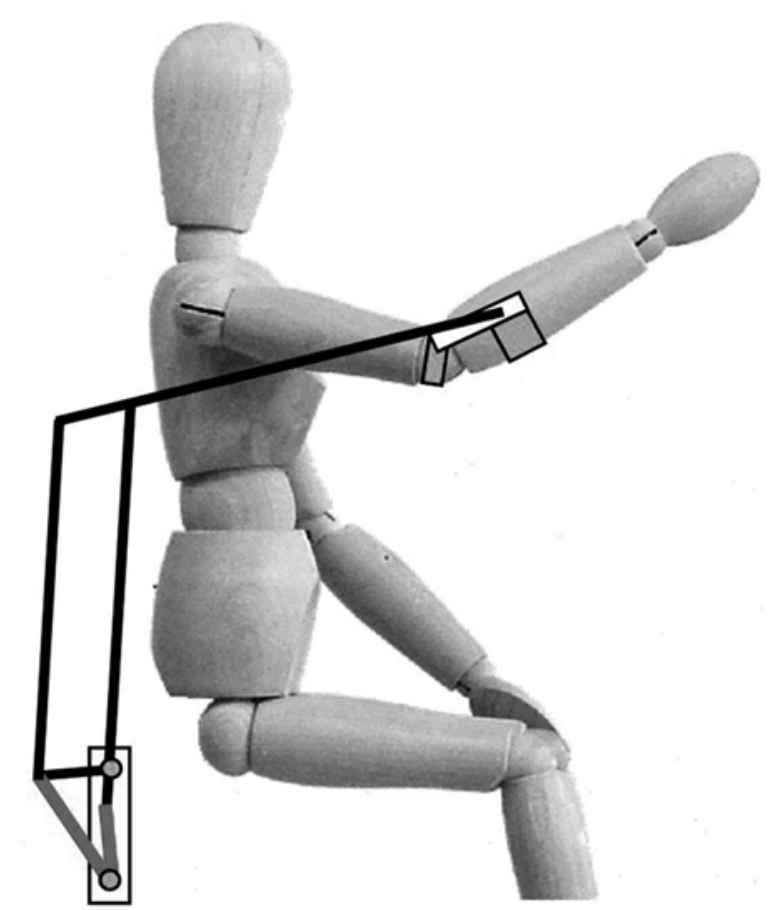

Figure 5.

Parallelogram linkage and balancer design used for second and third arm support prototypes: (a) diagram showing parameters and (b) diagram showing use as arm support. $m=$ supported mass, $k_{1}=$ stiffness of distal-link spring, $k_{2}=$ stiffness of proximal link spring, $L_{1}=$ distal link length, $L_{2}=$ proximal link length, $r_{1}=$ distal spring attachment arm length, $r_{2}=$ proximal spring attachment arm length, $a=$ vertical distance between fixed spring attachment and fulcrum. loss of balancing quality $[17,21]$, which we did in the final design.

From Equation (1), one can see that various options for adjustment of the balancer to varying supported mass: variation of fixed spring attachment point on the vertical $a_{i}$, variation of the spring attachment point on the links $r_{i}$, variation of the spring stiffness $k_{i}$, and variation of the link lengths $L_{i}$. From these, the first seems most practical. Another major advantage of placing the adjustment mechanism in the base is that a single adjustment mechanism is sufficient: under the condition that $a=a_{1}=a_{2}$ and if any inequality of link masses is compensated by an additional spring or counterweight, then adjustment of the fixed spring attachment $a$ provides simultaneous adjustment for both springs. This holds as long as Equation (1) and the following condition are met [22]:

$$
\frac{r_{1}}{L_{1}}=\frac{r_{2}}{L_{2}} .
$$

In our first prototype, which was not adjustable, we used a pulley-and-string arrangement to approximate the zero-free-length springs (Figure 6(a)) [2,21]. The maximum balancing error (static loads, not considering tissueinduced forces) was found to be about 5 percent relative to $23 \mathrm{~N}$ of user's arm weight and occurred in the configuration of reaching far forward [22]. In weak patients, the combination of the balancing error and friction proved too great, even though ball bearings were used throughout the mechanism. We furnished the second prototype with a different approximate balancer, in the form of wrapping cams with a rolling contact joint (Figure 6(b)) [22]. The resulting mean balancing error was about 3 percent with a maximum error of about 13 percent. Friction, however,
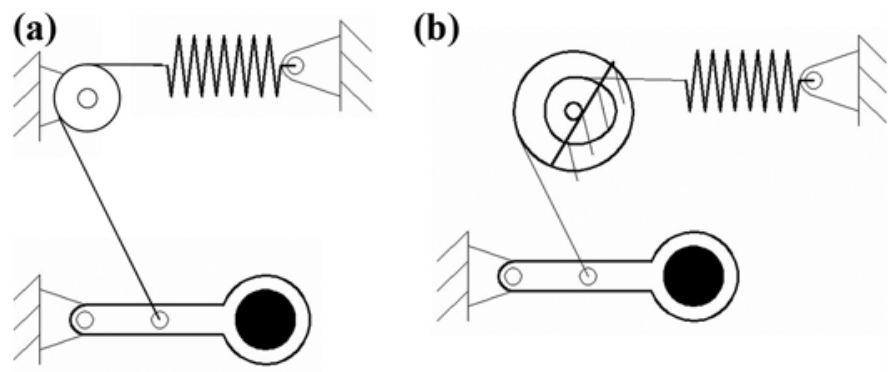

Figure 6.

Principle of gravity balancer: (a) pulley-and-string arrangement in first arm support prototype and (b) wrapping cams with rolling contact joint in second arm support prototype. 
was very low because of the rolling joints, which allowed many users to experiment with the device [13].

For the final design, we decided to employ another balancing principle, combining low friction with theoretically zero-balancing error. One special arrangement with multiple pulleys was suggested by Soethoudt (Figure 7) (cited in Herder) [20]. The principle is based on the emulation of an ideal spring by a pulley-and-string arrangement incorporating a normal spring (positive-free-length $l_{0}$ ), which is configured such that the string segments wrapped around the pulleys (of equal radius) add to (a multiple of) one pulley circumference for any position of the link. Consequently, the amount of wrapped string is constant. The parts of the string running parallel to the arms $a$ and $r$ are constant as well; hence, the part of the string running parallel to the connection line between the top roller center and the link roller center is the only part that is variable. Therefore, the spring elongation is equal to an imaginary zero-free-length spring elongation; hence, perfect balance is obtained, regardless of the pulley radius. Care must be taken to select the proper string length, which should be equal to

$$
L_{s}=a+r+2 \pi R-l_{0} \text {, }
$$

where $L_{S}$ is the string length, $R$ is the radius of the pulleys, and $l_{0}$ is the nonzero free length of the normal spring [20]. Depending on the desired ROM, spring selection in the arrangement according to Figure 7(a) can be difficult, (a)

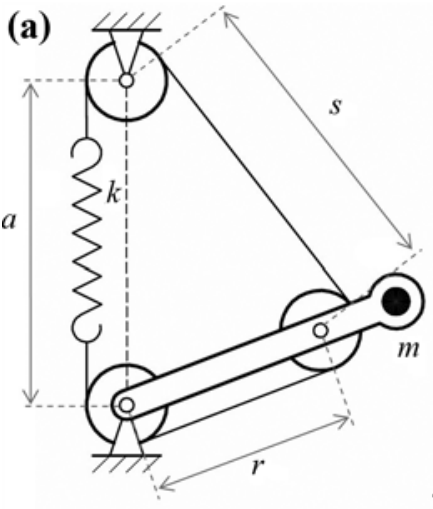

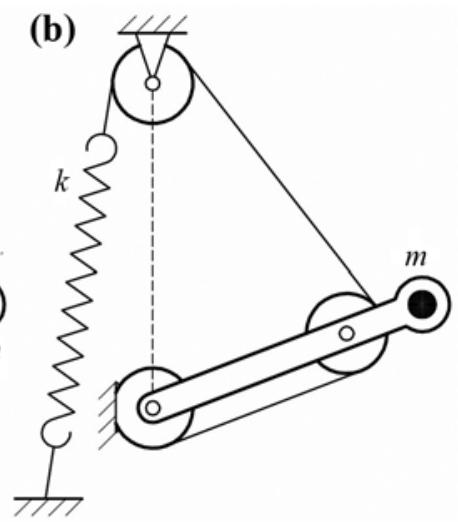

(b)
Figure 7.

Principle of balancing mechanism in third arm support prototype: (a) principal version with spring incorporated in string loop and (b) practical version with spring attached to frame (shown) or link. $a=$ effective vertical distance between fixed spring attachment and fulcrum, $r$ = effective spring attachment arm length, $s=$ effective spring extension, $m=$ supported mass, $k=$ spring stiffness. while the arrangement according to Figure 7(b) is most tolerant with respect to spring selection.

We applied the arrangement in Figure 7(b) in the arm support, where for each spring we incorporated one such pulley-and-string system in the design. The only difference with Figure 7(b) is that the springs are not fixed to the base but placed on their respective base links. A semi-see-through computer-aided design drawing is shown in Figure 8. The linkage architecture is identical to the one in Figure 5, but the zero-free-length springs are replaced with the pulley-and-string systems of Figure 7. The main advantages are that normal springs can be used without introducing a balancing error and that the simultaneous adjustment of both springs is maintained. When the mechanism is properly adjusted, the force needed at the interface to set the mechanism in motion (static loads, not

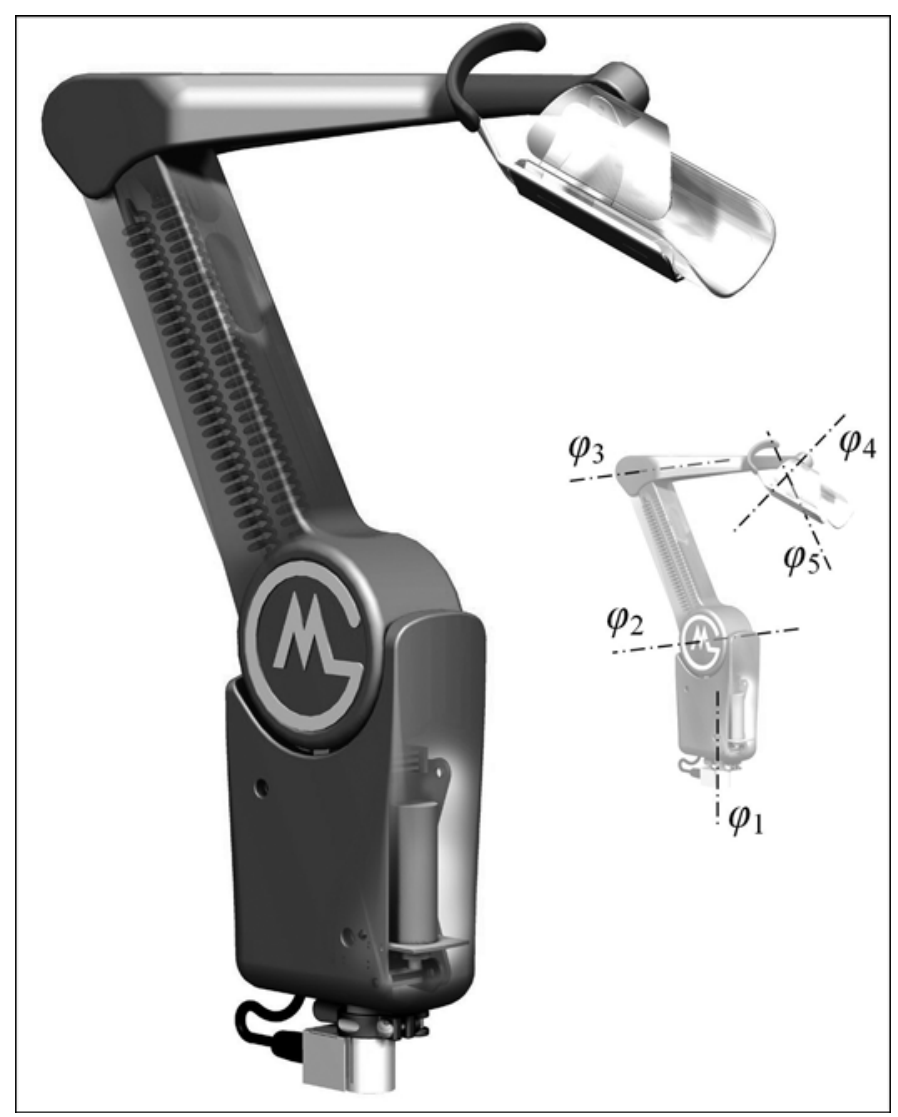

Figure 8.

Semi-see-through computer-aided drawing of third arm support prototype (Armon). Insert shows degrees of freedom $(\varphi)$ : $1=$ vertical base joint, 2 = fore/aft, 3 = up/down, 4 = interface horizontal axis, and 5 = interface vertical axis. 
considering tissue-induced forces) is around $0.2 \mathrm{~N}$, throughout the ROM, regardless of the load setting, and regardless of the direction of motion. This suggests that this "balancing error" is mainly due to friction, rather than the balancing mechanism itself.

\section{Improvement of Interface}

The importance of the interface cannot be overemphasized. It should be comfortable, be safe, and have a good feel. Other requirements for the interface are easy donning and doffing, avoiding shear forces, and allowing sufficient free skin for good perspiration. To an extent, the design of the complete device was guided by the interface requirements, starting with the CCM principle, which led to only a single interface brace. Shear forces were avoided by providing contact planes that together can generate the required support force $F_{\text {ccm }}$ while each of these components are normal to the support planes (Figure 9). A brace around the forearm and a curved segment behind the elbow are sufficient for this. For easy donning and doffing, the brace was composed of a top and a bottom section. The bottom section is designed to be thin and to allow some skin contact, particularly at the elbow, between the user and the armrest for feedback. The forearm is to be placed in the bottom section of the interface, while the top section closes automatically by the weight of the forearm and can be secured with Velcro.

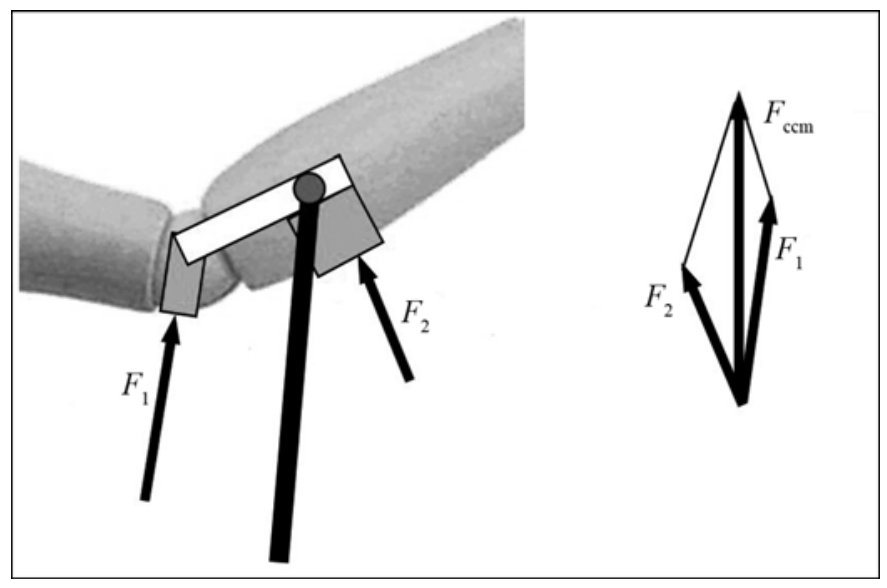

Figure 9.

Force system on interface of arm support: support force is resolved in two normal forces to avoid shear. $F_{1}=$ normal interface force on upper arm, $F_{2}=$ normal interface force on forearm, $F_{\mathrm{ccm}}=$ resultant vertical support force.

\section{General Improvement of Appearance}

The greatest source of inconspicuousness is the concept itself. Because no parts of the mechanism are around the shoulder or alongside the upper arm, the springs are hidden in the base links, and only one interface contact point is required, all parts contribute to a pleasing appearance. The second prototype was designed for a strong and powerful appearance, made up of closed and open elements that become more organic as the elements are located closer to the human body; however, the design goal was that the mechanism should be appealing yet inconspicuous [13]. The third prototype has a highly reduced box size because of the placement of the springs on the base links rather than in the box. Consequently, the box is smaller, the base links gained volume, and the device as a whole now has a gradual tapering from base to interface, which gives it a natural look (Figure 8).

\section{Ease of Use}

In principle, a passive balancer requires no separate control. It should create a zero-gravity sensation at all times. However, changing circumstances, e.g., putting on a coat or picking up an object, raise the user's desire to adjust the balancing settings. We decided, at the cost of increased complexity, to incorporate an electric motor that allows the user to adjust the balance. This motor is controlled by two low-operating-force switches on the wheelchair control unit. Pressing a first switch will increase the support force by a certain amount, whereas pressing a second switch will decrease the support force.

We needed to be able to tune the balancer down to a support force of zero (i.e., the springs only balance the weight of the mechanism itself). This allows users to "switch the device off," i.e., fixate the mechanism relative to the wheelchair. In preliminary trials, we found that the users sometimes perceive continuous pressure generated by the arm support as tiring. Moreover, to control the wheelchair by the joystick on uneven ground (e.g., a sidewalk), the mechanism needs friction between the user's forearm and the fixed armrest of the wheelchair. Diminishing the support force is then necessary, otherwise the weight of the arm does not rest on the fixed armrest; hence no friction is generated. To further eliminate undesired mechanism motion, we incorporated a friction brake that is automatically engaged as the balancer adjustment approaches zero, for instance, to avoid a floating arm when riding the wheelchair. 
Another mechatronic feature we incorporated is an automatic leveling function. A prerequisite for the balancing mechanism is to have a vertical base; otherwise, the mechanism will get one equilibrium position instead of a whole range. The leveling device automatically compensates up to $5^{\circ}$ of floor skew in any direction. This device can be overruled by two electric switches: one for tilting the device forward and one for tilting it backward. This can be used to generate force in the respective directions, for instance, to press an elevator button or work against contractures. Furthermore, users can switch the automatic leveling device off when, for instance, riding in a taxi van. A third switch is available that can be used to return to the nominal setting. All five switches are incorporated in a control unit: two switches for the balancer, two for the leveling device, and one to return the leveling device to default settings (Figure 10).

\section{RESULTS}

We designed and manufactured the arm support incorporating all the features described in the preceding section and called it "Armon" (coined by one of the users based on the first five letters of the Dutch word for arm support). The technical performance of the arm support is summarized in Table 1. The balancing quality and range exceed the requirements. The ROM is in accordance with the design specifications, with slight differences depending on the user. All users can reach their faces and laps or wheelchair tables. Figure $\mathbf{1 1}$ shows the Armon in key positions, while Figure 12 shows the limits of up and

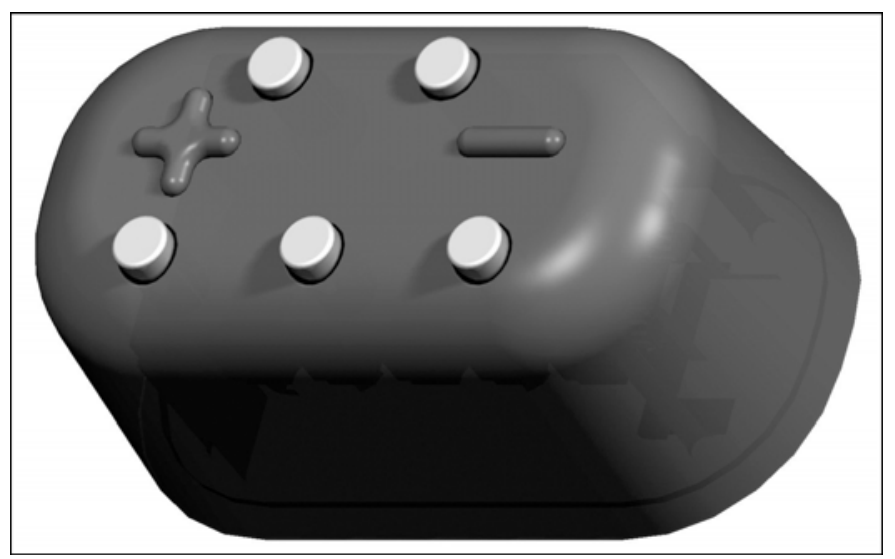

Figure 10.

Control unit with five switches to adjust balancer settings of arm support.
Table 1.

Design specifications and performance of Armon arm support.

\begin{tabular}{|c|c|c|}
\hline Requirement & $\begin{array}{c}\text { Design } \\
\text { Specification }\end{array}$ & Performance \\
\hline Balancing Range (N) & Up to $35 \mathrm{~N}$ & $\begin{array}{l}0-45 \mathrm{~N} \text {, where it is } \\
\text { possible to engage } \\
\text { friction brake at } 0 \mathrm{~N}\end{array}$ \\
\hline $\begin{array}{l}\text { Maximum Balancing } \\
\text { Error, Including } \\
\text { Friction (N) }\end{array}$ & $1 \mathrm{~N}$ & $0.2 \mathrm{~N}$ \\
\hline Range of Motion & - & $\begin{array}{l}\text { Base link: }-45^{\circ} \text { to } 25^{\circ} \\
\text { (aft/fore) relative to } \\
\text { vertical; Final link: max } \\
-50^{\circ} \text { to } 50^{\circ} \text { (down/up) } \\
\text { relative to perpendicu- } \\
\text { lar to base link }\end{array}$ \\
\hline
\end{tabular}

down movements. More specifically, the workspace in terms of hand position, although depending on the user's specific anthropometry, ranges in down/up direction from well below the fixed armrest (Figure 12(a)) to forehead level (Figure 12(b)). Sideways in/out movement is restricted only by interference of the arm with the trunk, effectively allowing the user to move across his or her lap or wheelchair table, while moving out laterally is restricted only by the natural constraints of the user's arm. In addition, the elbow can be moved aft/fore over the entire length of the fixed armrest.

Potentially, interference with a large backrest of the wheelchair can occur if the device is moved out laterally from a position where the elbow of the user is far back. This situation does not occur in practice (Figure 12(a)), probably because users are accustomed to rotating the whole wheelchair to face the activities.

The device does not require added clearance in any direction. If the forearm is directed straight forward, then the curve in the final link protrudes around $50 \mathrm{~mm}$ lateral to the fixed armrest of the wheelchair. However, if the hand is moved slightly inward (i.e., the forearm rotates in a horizontal plane), the device is completely inside the wheelchair contours.

The mass of the device is $5 \mathrm{~kg}$. It can be easily mounted and dismounted: after releasing a plug (controls and power feed) and a quick-detachable coupling, the device can be lifted off the wheelchair. Mounting is just as easy, in reverse order. All personal adjustments will be maintained. The device can be mounted on a variety of powered wheelchairs, except very small ones for children. 


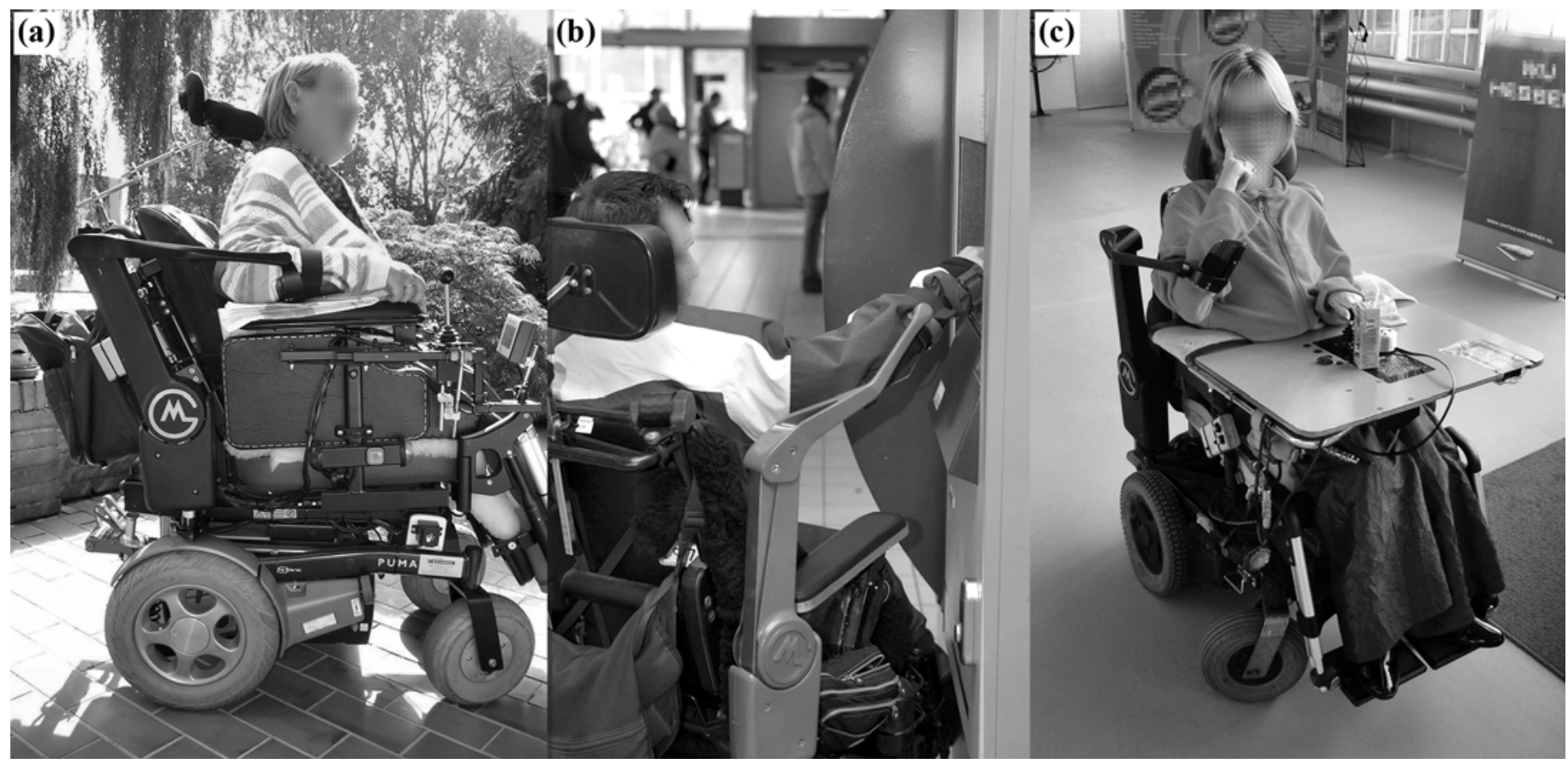

Figure 11.

Armon arm support in use: (a) nominal position, (b) reaching forward, and (c) touching one’s face.

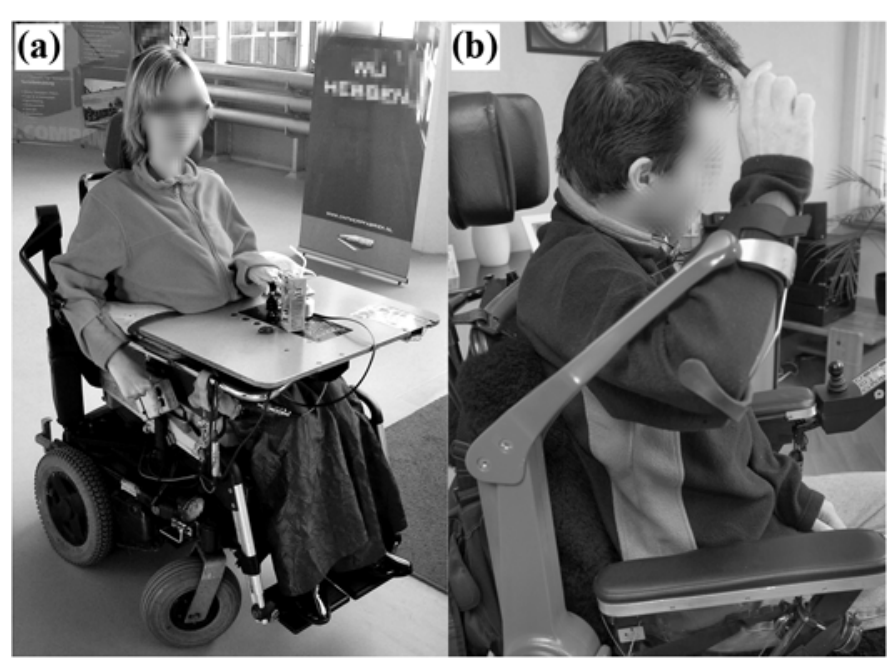

Figure 12.

Extreme down and up positions of Armon arm support: (a) lower limit and (b) upper limit.

The device can also be mounted to normal or desk chairs. Fitting it to a manual chair would require the installation of a battery, and the large rear wheel may obstruct optimal placement.

Initial fitting at the rehabilitation center mainly concerns the fixation at the proper location on the wheel- chair, wiring the electric control cables, and adjusting the interface to the user's forearm shape.

So far, no formal user evaluation studies have been performed. The device has become available only recently, and five people used it for 2 months. In addition, two people have been fitted with beta versions of the design continuously for about 6 months. The main difference with the final version is that one person (user 2) did not have the leveling device installed. Table 2 summarizes the characteristics of three of the users and their use of the arm support.

The users listed in Table 2 employ the arm support continuously. Others use the device only at home for eating, drinking, and keyboarding. The main findings of the

Table 2.

Overview of users on right side of arm support.

\begin{tabular}{lcll}
\hline \multirow{2}{*}{\multicolumn{1}{c}{ Characteristic }} & \multicolumn{3}{c}{ User } \\
\cline { 2 - 4 } Sex & Female & Female & Male \\
Disease & SMA I & SMA II & Becker \\
Prior Experience & Manus & Top Help & None \\
Duration of Use (mo) & 6 & 6 & 2 \\
Daily Use (h/day) & 10 & 8 & 6 \\
\hline SMA = spinal muscular atrophy. & & & \\
\hline
\end{tabular}


users are given next, grouped according to the five areas for improvement identified in the "Final Design" section.

\section{Range of Motion: Functionality}

The general opinion on the functionality is very positive. User 1 reports that with the Armon, she can put on her glasses, apply her makeup, and tickle her nose. Furthermore, she is able to drink independently (directly from a cup, not with a straw), operate lift buttons, and prepare food in a microwave oven. Important for her is that she can eat precut food independently: she no longer needs to be fed when eating pizza out with friends. Also, she can take medication by herself. She reports no interference of the mechanism with the wheelchair or her arm, but she cannot reach far out to the side. User 2 is relieved that she is, for the first time in years, able to eat and drink independently. She emphasizes that it is very difficult for someone else to perform elementary intimate tasks such as putting on her glasses (the result is that the glasses are skewed, against her eyelashes, or fingerprinted) or scratching, and she is happy do be able to do this herself now. She finds the ROM sufficient in vertical direction, but she is not able to reach out to the side further than just enough to let her arm hang down next to the wheelchair, comparable with user 1 (Figure 12(a)). Several ADL have become possible, including raising a glass (toast), picking up her mail from the mailbox, and shaking hands, which is important to her. User 3 uses the Armon to independently operate a public cash machine, play chess, smoke, and comb his hair.

\section{Balancing Quality}

User 1 is pleased that the Armon has no perceivable friction, as the first prototypes had. A small push is enough to set her arm in motion. Because of her muscular weakness, she frequently uses the adjustment function, although the nominal setting provides sufficient balance to allow her to move without readjustment. User 2 remarks that she can perform goal-directed movements accurately and securely, which she could not do with other products. Because people hardly notice that she is using an aid, she concludes that the device must provide a natural motion pattern.

\section{Interface}

User 1 does not need the top section of the forearm brace. Indeed, with the top section installed, she encounters more resistance due to friction with clothing. Even when the forearm comes loose from the brace, safe contact is maintained through the elbow segment (Figure 11(c)). Only when reaching down to the switches located at the side of the wheelchair (Figure 12(a)) is she at risk of sliding out of the interface. Yet she manages and would rather not have the top section installed. Thanks to the absence of the top section, she can put her arm in or out of the interface by herself. She locks the interface next to the fixed armrest, slightly lower, and then drops her arm from the armrest into the interface. By placing the wheelchair next to a wall or anything fixed, she makes sure that the mechanism does not move out to the side. User 2 notes that proper fitting of the interface is critical, otherwise either the elbow or the hand drops down. Furthermore, she reports some skin irritation when used with sleeveless clothing. The Velcro attachment of the top section sometimes damages wool sweaters.

\section{Aesthetics}

The general appearance is widely and highly appreciated. User 1 reports several occasions during which people did not notice the arm support until after half an hour. User 2 also finds it inconspicuous and pleasing, which is important for her. The top link of the arm support makes her wheelchair somewhat wider. Therefore, she has the arm support removed when she is in buildings with normal-sized doors. Hitting the post may push her forearm forward, which is potentially problematic because she operates her wheelchair with the same arm. One of the 2-month users used to have two suspension-type arm support devices (strings from an overhead pulley construction) and now has two Armons. He reports that people used to first see the devices and then him, but that is now inverted, much to his pleasure.

\section{Ease of Use}

Since no separate control is needed for the motion, the users can work with the device almost immediately. Initially, they still work primarily with their body, but gradually they learn to do more and more with their arms by themselves, thus continually discovering new possibilities. Also, the operation of the switches requires little learning. In approximately a day, the users became accustomed to operating the switch unit. User 2 remarks that she at first used the balancer adjustment much more than she does now, as she relaxes her whole body more and more when moving her arm with the device. The brake, which is engaged when the support force is trimmed 
completely down, is used for wheeling outside, when traveling by taxi or train, and sometimes at home as well.

\section{DISCUSSION}

The presence of the mechatronic functions places the Armon between the categories of passive orthosis and powered orthosis. However, the fact that the device is essentially passive implies that no motion control input device is required, such as separate robotic arms or powered orthoses. Furthermore, because actuators do not drive the user's arm motion, the device is inherently safe. An added advantage is that the device is silent. For instance, in an office or at a dinner table, the control motors only run at the user's command.

The preliminary results give rise to some observations. The users became familiar with the device in about a day. Some reported that at first it felt a bit insecure to break the contact between the elbow and the wheelchair's fixed armrest ("loose terra firma"), because the armrest is used to maintain trunk balance. However the elbow is easy to move back to the armrest if desired. Usually within the first day, users discover that the trunk motion they used to employ to aid arm motion is no longer needed. From that point on, they truly start "letting the device do the work.”

The device was intended for people classified by the Brooke index between categories 3 and 5. One of our subjects (user 1) is, in fact, weaker but proved able to the use the device effectively. This is probably because the balancing error is smaller than initially demanded. For nondisabled persons, a support force threshold of $0.2 \mathrm{~N}$ of force (including friction), roughly corresponding to the weight of a penlight battery, is hardly perceptible relative to the support force. However, to user 1, it cannot be low enough. This user uses the controls extensively (adjusting and tilting) and does so hardly without noticing ("it goes automatically"). The other beta version user (user 2) is looking forward to receiving the tilting function, in particular for keyboarding, because she cannot reach far enough forward for longer times.

Why users find that actively moving their arm out to the side is difficult is not quite clear. The ROM does not restrict this or reduce the balancing quality in that range. The difficulty may be partly explained by contractures or other physiological phenomena that generate more resistance in that range. Another factor may be that users are not used to actively moving their arms in that range. Nevertheless, all users mention that they move much more than they used to, and yet, apart from the first few days, this does not exhaust them at all.

Proper fitting of the device to the user's arm and the wheelchair is critical. If the interface is not properly located, either the elbow or hand will drop. If the interface is not properly adjusted to the user's arm shape, the interface will move up or down, with the same effect. Deviations of $5 \mathrm{~mm}$ from the CCM can make the difference of floating or dropping. If carefully aligned, the Armon allows people as weak as user 1 to use the device. In the interface of the Armon, the bottom part is not crucial to precise balancing, but the part behind the elbow is. This part supports a relatively stiff part of the arm. Only skin and tendons are between the interface and the bone, which is sufficiently stiff for the required adjustment accuracy. The interface is designed such that the arm does not slip: it is supported by normal forces only. Correct adjustment is thus maintained while the arm is moved around.

Also, the location of the fixed point on the wheelchair is crucial. If the base is located on or near the vertical through the user's shoulder, then the mechanism can reach a singularity if the interface approaches this line. The mechanism was designed to have sufficient ROM when the base is placed about $0.1 \mathrm{~m}$ from the vertical through the shoulder to the rear of the wheelchair (Figure 11(a)). This will ensure smooth and easy motion.

Users 1 and 3 have found functionality that was not anticipated during the design. With the arm support balancing nominally, they move their arms up and then hold their arms up by clamping a finger between their teeth while lowering the balancer setting until the brake engages. They can then let go of their finger and lean on the device, as if it were a raised fixed armrest. User 1 does this occasionally for the pleasant feel of it and for blowing her nose. User 3 uses this to support his elbow at a higher level that allows him to play darts.

\section{CONCLUSIONS}

We present this spring-balanced MAS for people with muscular weakness based on the biomechanical principle that complete static balance is achievable with a single interface location by using the observation that the shoulder joint can carry part of the arm weight. While 
single-interface arm supports exist, this analysis led to a novel conceptual design, consisting of a parallelogram mechanism with two springs. A special pulley-and-string arrangement was used to combine low friction with virtually zero balancing error and adjustment of the support force without loss of balancing quality. The level of the support force can be adjusted by a switch-operated electromechanical system, which is useful when the load changes (putting on an coat, picking up object) or when a vertical force is to be generated (pressing buttons, overcoming friction between arm and body or clothing). Another mechatronic function is a leveling device, which keeps the base of the balancer upright. Also, this device can be overruled to generate horizontal force (reaching far forward or backward) or to switch the function off (wheeling on uneven ground, riding in a taxi). These mechatronic functions only adjust the balancer: the main motion generator is the user; therefore, the device is inherently safe. Several users appreciate the functionality and appearance. They have gained independence and no longer rely on assistance for many ADL. As the device becomes more widely available, formal user assessments will be conducted. Furthermore, we intend to conduct a detailed qualitative and quantitative comparison with other MAS designs.

\section{ACKNOWLEDGMENTS}

The MAS presented in this article is the result of contributions of a variety of persons. We gratefully acknowledge the Dutch Association for Neuromuscular Diseases, in particular J. F. Jordaans, for its enthusiasm and support. We owe a large debt of gratitude to the persons kind enough to receive us for extensive home visits and interviews and to their physician, I. J. M. de Groot, MD, from the Maastricht Academic Medical Center and Rehabilitation Center De Trappenberg. Furthermore, a number of master of science thesis works have been devoted to aspects of the design, in particular those of Sergio Tomazio, Luis Cardoso, Jorine Koopman, Clara Gil Guerrero, Wendy van Stralen, Sabine Gal, and Pieter Lucieer, who were all supervised by J. Herder. The final design stage was largely sponsored by Microgravity Products (MGP), which currently produces the Armon. We gratefully acknowledge the rehabilitation center Dorp Rehabilitation Technology for distributing and fitting the arm support to the users and their wheelchairs.
This work was unfunded at the time of manuscript preparation.

The authors are named as inventors on a patent on the device that is under application, while all but J. Herder are employed by MGP. Consequently, some of the authors might potentially have a conflict of interest. However, all quantitative data were measured objectively; all user opinions were recorded sincerely while drafts of this article were presented to them for verification prior to publication. We therefore believe that the article gives a genuine and honest account of the present state of the Armon device.

\section{REFERENCES}

1. Munsat TL. The spinal muscular atrophies. Curr Neurol. 1994:14(3):55-71.

2. Cardoso LF, Tomazio S, Herder JL. Conceptual design of a passive arm orthosis. Proceedings of the American Society of Mechanical Engineering (ASME) Design Engineering Technical Conference, MECH-34285; Montreal, Canada; 2002 Sep 29-Oct 2. New York (NY): ASME; 2002.

3. Mahoney RC. Robotic products for rehabilitation: Status and strategy. In: Proceedings of the 5th International Conference on Rehabilitation Robotics; 1997 Apr 14-15; Bath, United Kingdom.

4. Johnson GR, Buckley MA. Development of a new Motorized Upper Limb Orthotic System (MULOS). Proceedings of the Rehabilitation Engineering and Assistive Technology Society of North America (RESNA); 1997 Jun; Pittsburgh, PA. Arlington (VA): RESNA; 1997. p. 399-401.

5. Landsberger S, Leung P, Vargas V, Shaperman J, Baumgarten J, Yasuda LY, Sumi E, McNeal D, Waters R. Mobile arm supports: History, application, and work in progress. Top Spinal Cord Inj Rehabil. 2005;11(2):74-94.

6. Homma K, Arai T. Upper limb motion assist system with parallel mechanism. J Rehabil Soc Jpn. 1995;15(1):90-97.

7. Chyatte SB, Vignos PJ. The balanced forearm orthosis in muscular dystrophy. Arch Phys Med Rehabil. 1965;46(9): 633-36. [PMID: 5828903]

8. Skorecki J. Biomechanical applications of the synthesis of mechanisms. Proceedings of the 3rd IFToMM World Congress. Vol. E. 1971; Kupari, Yugoslavia. p. 311-22.

9. Rahman T, Sample W, Seliktar R, Alexander M, Scavina M. A body-powered functional upper limb orthosis. J Rehabil Res Dev. 2000;37(6):675-80. [PMID: 11321003]

10. JAECO Orthopedic [home page on the Internet]. Hot Springs (AR): JAECO Orthopedic; c1955-2006 [updated 2006; cited May 11, 2006]. JAECO mobile arm support; [1 screen]. Available from: http://www.jaeco-orthopedic.com. 
11. Focal Revalidatietechniek [home page on the Internet]. Berkel-Enschot (the Netherlands): Focal Revalidatietechniek; c2005-6 [updated 2006; cited May 11, 2006]. Focal TOP HELP; [about 2 screens]. Available from: http:// www.focalmeditech.nl/.

12. Brooke MH. Functional evaluation and grading system for neuromuscular patients: a clinician's view of neuromuscular diseases. Philadelphia (PA): Lippincott Williams \& Wilkins. p. 30-33.

13. Herder JL. Development of a statically balanced arm support: Armon. Proceedings of the 9th International Conference on Rehabilitation Robotics; 2005 Jun 28-Jul 1 Chicago, IL. p. 281-86.

14. Stanger CA, Anglin C, Harwin WS, Romilly DP. Devices for assisting manipulation: A summary of user task priorities. IEEE Trans Rehabil Eng. 1994;2(4):256-65.

15. Harwin, WS, Rahman T, Foulds RA. A review of design issues in rehabilitation robotics with reference to North American research. IEEE Trans Rehabil Eng. 1995;3(1):3-13.

16. Rahman T, Stroud S, Ramanathan R, Alexander M, Seliktar $\mathrm{R}$, Harwin W. Task priorities and design for an arm orthosis. Technol Disabil J. 1996;5(2):197-203.

17. Herder JL, Tuijthof GJM. Two spatial gravity equilibrators. Proceedings of the Design Engineering Technical Conferences and American Society of Mechanical Engineers
(ASME) Computers and Information in Engineering Conference; 2000 Sep 10-13; Baltimore, MD. New York (NY): ASME; 2000.

18. Carwardine G. Improvements in equipoising mechanism. UK Patent 433.617. 1935.

19. Streit DA, Shin E. Equilibrators for planar linkages. J Mech Des. 1993;115(3):604-11.

20. Herder JL. Energy-free systems. Theory, conception and design of statically balanced spring mechanisms [dissertation]. Delft (the Netherlands): Delft University; 2001.

21. Soper R, Mook D, Reinholtz C. Vibration of nearly perfect spring equilibrators. Proceedings of the 1997 American Society of Mechanical Engineers (ASME) Design Engineering Technical Conference, DAC-3768. New York (NY): ASME; 1997.

22. Lucieer P, Herder JL. Design of an adjustable compensation mechanism for use in a passive arm support. Proceedings of the American Society of Mechanical Engineers (ASME) Design Engineering Technical Conferences, DETC2005-85442; 2005 Sep 24-28; Long Beach, CA. New York (NY): ASME; 2005.

Submitted for publication May 11, 2006. Accepted in revised form September 7, 2006. 